\title{
Novel FGFR3 mutations creating cysteine residues in the extracellular domain of the receptor cause achondroplasia or severe forms of hypochondroplasia
}

Solange Heuertz, Martine Le Merrer, Bernhard Zabel, Michael Wright, Laurence LegeaiMallet, Valérie Cormier-Daire, Linda Gibbs and Jacky Bonaventure

European Journal of Human Genetics (2006) 14, 1321. doi:10.1038/sj.ejhg.5201745

Correction to: European Journal of Human Genetics (2006) 14, 1240-1247. doi:10.1038/sj.ejhg.5201700, published online August 16, 2006
Since the above paper has been published online the authors have identified some errors in the Nucleotide change column of Table 2 . The corrected table is shown below.

Table 2

\begin{tabular}{|c|c|c|c|c|c|c|}
\hline Patient & Familial/sporadic & Nucleotide change & Exon & Amino-acid substitution & Domain & Phenotype \\
\hline 1 & $S$ & $835 A>T$ & 7 & S279C & Ig IIIa & $\mathrm{ACH}$ \\
\hline 2 & $S$ & $833 A>G$ & 7 & Y278C & Ig IIIa & $\mathrm{HCH} / \mathrm{ACH}$ \\
\hline 3 & $\mathrm{~S}$ & $802 \mathrm{G}>\mathrm{T}$ & 7 & G268C & lg II-lglll linker & $\mathrm{HCH}$ \\
\hline 4 & $\mathrm{~S}$ & $598 \mathrm{C}>\mathrm{T}$ & 5 & R200C & $\lg 11$ & $\mathrm{HCH}$ \\
\hline 5 & $\mathrm{~S}$ & $784 A>C$ & 7 & $\mathrm{~N} 262 \mathrm{H}$ & Ig II-Iglll linker & $\mathrm{HCH}$ \\
\hline 6 & $\mathrm{~F}$ & $251 \mathrm{C}>\mathrm{T}$ & 3 & S84L & $\lg 1$ & $\mathrm{HCH}$ \\
\hline 7 & $\mathrm{~S}$ & $1142 \mathrm{~T}>\mathrm{A}$ & 10 & V381E & TM & $\mathrm{HCH}$ \\
\hline
\end{tabular}

\section{ERRATA}

\section{A test of homogeneity of Hardy-Weinberg disequilibrium across strata}

Xiao-Lin Yin, Wen-Qing Ma, Man-Lai Tang and Jianhua Guo

European Journal of Human Genetics (2006) 14, 1321. doi:10.1038/sj.ejhg.5201746

Correction to: European Journal of Human Genetics (2006) 14, 1223-1230. doi:10.1038/sj.ejhg.5201689

Due to a typesetting error a formula in Appendix B was published incorrectly.
In Appendix B, the formula $I_{k}=\left(D_{k} I_{k D_{k} p_{k}} I_{k p_{k} D_{k}} I_{k p_{k} p_{k}}\right)$ should be changed as

$$
I_{k}=\left(\begin{array}{ll}
I_{k D_{k} D_{k}} & I_{k D_{k} p_{k}} \\
I_{k p_{k} D_{k}} & I_{k p_{k} p_{k}}
\end{array}\right)
$$

